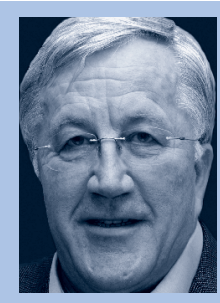

Prof. Dr. rer. nat. med. habil. Rainer Breul D.O. h.c.
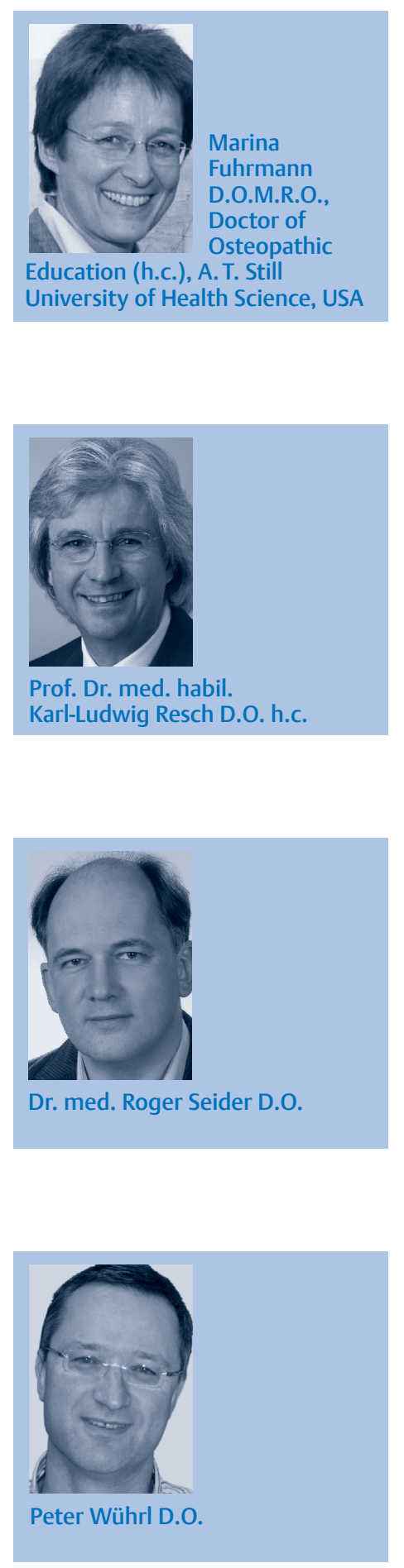

\section{Fortschritt und Idyll}

$\mathrm{M}$ anchmal scheint es einem, als wäre die osteopathische Praxis der letzte verteidigungswerte Ort im Gesundheitswesen, ein Idyll für Patienten und Therapeuten gleichermaßen. Fern der Zeittakte, Diagnoseschlüssel und Abrechnungsnummern lässt sich noch Medizin mit und für die Patienten betreiben. Diagnostischer Aufwand und therapeutischer Anspruch stehen noch in einem vertretbaren Verhältnis und die individuelle, subjektiv wahrgenommene Realität des Patienten darf berücksichtigt werden. Mit Recht könnten wir zudem beanspruchen, dass unser Handeln praktische Wissenschaft am besonderen Fall ist, dem jeweils singulären Leiden des unsere Hilfe suchenden Patienten.

Die Therapeuten genießen dieses Idyll zu Recht. Während andere Berufsgruppen sich in immer feineren Spezialisierungen verlieren, ist es Aufgabe und erklärtes Ziel der Osteopathen, die Ganzheit zu bewahren. Während andere bis zu einem Drittel ihrer Arbeitszeit mit bürokratischen Dokumentations- und Abrechnungspflichten verbringen, sind wir frei, hautnah an den Anliegen unserer Patienten zu arbeiten und müssen bei der Diagnosestellung noch nicht einmal die neuen Rechtschreibregeln einhalten. Hier wird ein Zipfel der Gefahr greifbar, dass idyllische Abgeschiedenheit in illusorische Isolation umschlagen kann.

Der allgemeine Vorschuss an Vertrauen und Interesse (oder Skepsis und Misstrauen), der den Osteopathen entgegengebracht wird, ist weder begründet noch begründbar. Auch Osteopathen sind Menschen, meist handeln sie verantwortungsvoll, sind aber zuweilen auch lausige Therapeuten - ganz wie andere Berufsgruppen. Die These, dass die Anwendung der Osteopathie die sie anwendenden Osteopathen menschlich veredelt, ist gewagt und sollte gewiss nicht von uns selbst entschieden werden. Dagegen ist Fortschritt und Veredelung der Osteopathie als verantwortliches Handeln unsere ureigenste, osteopathische Aufgabe; und es ist fraglich, ob diese gelingen kann, wenn wir uns allzu behaglich in unserem Idyll eingerichtet haben. Fortschritt mag ein belastetes Wort sein, aber die fortschreitende Entwicklung der osteopathischen Konzepte und die Verbreiterung der osteopathischen Versorgung sind für das Bestehen einer so jungen Praxis und Wissenschaft grundlegend. Behagliches Zurücklehnen angesichts der beeindruckenden Behandlungserfolge ist berechtigt, aber rechtfertigt keineswegs die Abschottung gegenüber dem wissenschaftlichen Diskurs und der impliziten Kritik, die in anderen Sichtweisen ausgedrückt ist.

Symptome dafür, dass diese Abschottung zum Nachteil der Osteopathie geschieht, gibt es genug: Grundlegende Konzepte und Begriffe werden von verschiedenen Osteopathen oft völlig unterschiedlich angewandt, sodass eine sinnvolle Konversation erst möglich ist, wenn man sich gemeinsam durch den Begriffswirrwarr hindurch definiert hat; unsere Begriffe stehen zuweilen - bei gleicher Wortwahl - im Widerspruch zu anderen Wissensbereichen, ohne dass diese Diskrepanz und osteopathische Eigensinnigkeit offen ausgedrückt und begründet werden. Auch das Kardinalsymptom einer sich selbst ungewissen und den eigenen Fähigkeiten wenig vertrauenden Wissenschaft ist zu finden: Autorität geht vor Argument. Dabei ist es ja gerade das gut vorgetragene und überzeugende Argument, das wissenschaftliche Autorität begründet. Und was verbürgt Autorität im Bereich der praktischen Wissenschaft? Der Heilungserfolg? Sicherlich, aber auch das offene Nachdenken über die Bedingungen des Erfolgs und Misserfolgs. Das ist zugleich der Blick auf uns selbst in dem beneidenswerten Idyll, der uns davon abhalten kann in bedauerliche Isolation zu verfallen.

Die Herausgeber

Titelbild: Oben: Neugeborenen-Sphenoid. Aufnahme: Prof. Dr. R. Breul, Anatomisches Institut der LMU, München. Unten: Sphenoid eines eineinhalbjährigen Kindes. Aus: Bardeleben, K von: Handbuch der Anatomie des Menschen. Erster Band: Skeletlehre. Jena:

Fischer; 1896. Bearbeitung Pdesign, Stuttgart 2008. 